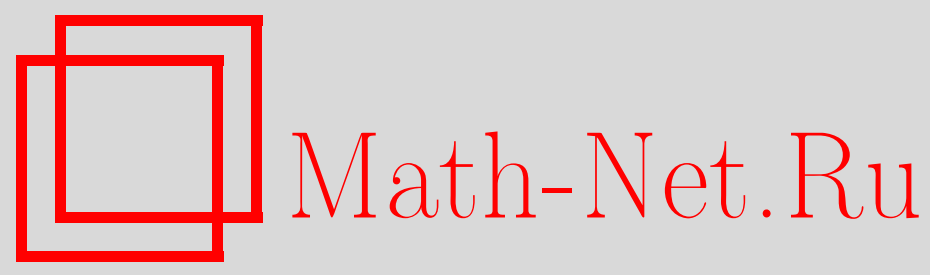

В. А. Быковский, Теорема Валена для двумерных подходящих дробей, Матем. заметки, 1999, том 66, выпуск 1, 30-37

DOI: https://doi.org/10.4213/mzm1139

Использование Общероссийского математического портала Math-Net.Ru подразумевает, что вы прочитали и согласны с пользовательским соглашением http://www. mathnet.ru/rus/agreement

Параметры загрузки:

IP: 3.85 .73 .92

26 апреля 2023 г., 14:13:54

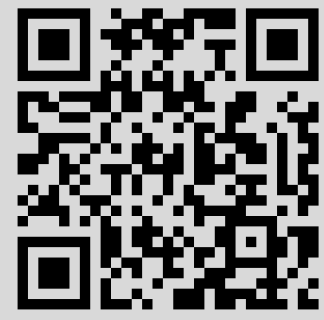




\section{ТЕОРЕМА ВАЛЕНА ДЛЯ ДВУМЕРНЫХ ПОДХОДЯЩИХ ДРОБЕЙ}

\section{В. А. Быковский}

В статье изучаются некоторые свойства относительных минимумов трехмерных решеток и совместные диофантовы приближения для двух вещественных чисел.

Библиография: 3 названия.

Введение. Пусть $\alpha=\left[d_{0}, \ldots, d_{i}, \ldots\right]$ - разложение вещественного $\alpha$ в цепную дробь и

$$
\frac{a^{(i)}}{q^{(i)}}=\left[d_{0}, \ldots, d_{i-1}\right]
$$

- подходящая дробь с НОД $\left(a^{(i)}, q^{(i)}\right)=1$. Существует несколько многомерных обобщений понятия "подходящая дробь". В работе мы будем изучать одно из самьх удачных, предложенное независимо Вороным и Минковским (см. [1]).

Пусть $\alpha_{1}, \ldots, \alpha_{t} \in \mathbb{R}^{t}, q$ - натуральное число и $a_{1}, \ldots, a_{t}$ - ближайшие целые к $\alpha_{1} q, \ldots, \alpha_{t} q$ соответственно. Назовем выражение $\left(a_{1}, \ldots, a_{t}: q\right)$ подходящей дробъю первого типа к $\left(\alpha_{1}, \ldots, \alpha_{t}\right)$, если для всех других натуральных $q^{\prime}<q$ не вьполняются одновременно $t$ неравенств

$$
\left\|q^{\prime} \alpha_{1}\right\| \leqslant\left\|q \alpha_{1}\right\|, \ldots,\left\|q^{\prime} \alpha_{t}\right\| \leqslant\left\|q \alpha_{t}\right\|
$$

где $\|\alpha\|$ - расстояние от $\alpha$ до ближайшего целого. Целочисленньй вектор $\left(a_{1}, \ldots, a_{t}\right)$ назовем числителем, а натуральное $q$ знаменателем. Обозначим через $\Delta\left(\alpha_{1}, \ldots, \alpha_{t}\right)$ множество всех знаменателей $q$, которые однозначно определяют подходящие дроби. Это множество конечно тогда и только тогда, когда $\alpha_{1}, \ldots, \alpha_{t}$ - одновременно рациональные числа. При $t=1$ согласно хорошо известной теореме Лагранжа (см. [2] или [3]) получаем подходящие дроби из (0.1). По теореме Минковского о линейных формах для любого знаменателя $q \in \Delta\left(\alpha_{1}, \ldots, \alpha_{t}\right)$ выполняется оценка (см. [3])

$$
q\left\|\alpha_{1} q\right\| \ldots\left\|\alpha_{t} q\right\|<1
$$

Пусть $\left(\alpha_{1}, \ldots, \alpha_{t}\right) \in \mathbb{R}^{t},\left(q_{1}, \ldots, q_{t}\right) \neq(0, \ldots, 0)$ - набор цельх чисел и $a-$ ближайшее целое к $\alpha_{1} q_{1}+\cdots+\alpha_{t} q_{t}$. Назовем выражение $\left(a: q_{1}, \ldots, q_{t}\right)$ подходящей дробью второго типа $\mathrm{K}\left(\alpha_{1}, \ldots, \alpha_{t}\right)$, если для всех других целочисленных наборов $\left(q_{1}^{\prime}, \ldots, q_{t}^{\prime}\right) \neq$ $(0, \ldots, 0)$ с $\left|q_{i}^{\prime}\right| \leqslant\left|q_{i}\right|(i=1, \ldots, t)$ вьполняются неравенства

а) при $\left(\left|q_{1}\right|, \ldots,\left|q_{t}\right|\right) \neq\left(\left|q_{1}^{\prime}\right|, \ldots,\left|q_{t}^{\prime}\right|\right)$

$$
\left\|q_{1} \alpha_{1}+\cdots+q_{t} \alpha_{t}\right\|<\left\|q_{1}^{\prime} \alpha_{1}+\cdots+q_{t}^{\prime} \alpha_{t}\right\| ;
$$


б) при $\left(\left|q_{1}\right|, \ldots,\left|q_{t}\right|\right)=\left(\left|q_{1}^{\prime}\right|, \ldots,\left|q_{t}^{\prime}\right|\right)$

$$
\left\|q_{1} \alpha_{1}+\cdots+q_{t} \alpha_{t}\right\| \leqslant\left\|q_{1}^{\prime} \alpha_{1}+\cdots+q_{t}^{\prime} \alpha_{t}\right\| .
$$

Число $а$ назовем числителем, а вектор $\left(q_{1}, \ldots, q_{t}\right)$ - знаменателем. Обозначим через $\Delta^{*}\left(\alpha_{1}, \ldots, \alpha_{t}\right)$ множество знаменателей для подходящих дробей второго типа. Очевидно, что $\pm\left(q_{1}, \ldots, q_{t}\right)$ входят в $\Delta^{*}\left(\alpha_{1}, \ldots, \alpha_{t}\right)$ только одновременно и их можно не различать. С этой точки зрения при $t=1 \Delta\left(\alpha_{1}\right)=\Delta^{*}\left(\alpha_{1}\right)$.

Множество $\Delta^{*}\left(\alpha_{1}, \ldots, \alpha_{t}\right)$ конечно тогда и только тогда, когда $\alpha_{1}, \ldots, \alpha_{t}$ - одновременно рациональные числа. По теореме Минковского о линейных формах для любого знаменателя $\left(q_{1}, \ldots, q_{t}\right) \in \Delta^{*}\left(\alpha_{1}, \ldots, \alpha_{t}\right)$ вьполняется неравенство

$$
\left|q_{1} \ldots q_{t}\right|\left\|\alpha_{1} q_{1}+\cdots+\alpha_{t} q_{t}\right\|<1 .
$$

Согласно классической теореме Валена для двух соседних подходящих дробей вида (0.1) справедлива оценка (см. [2] или [3])

$$
\min \left\{q^{(i)}\left|a^{(i)}-\alpha q^{(i)}\right|, q^{(i+1)}\left|a^{(i+1)}-\alpha q^{(i+1)}\right|\right\}<\frac{1}{2},
$$

которую можно записать в виде

$$
\min \left\{q^{(i)}\left\|\alpha q^{(i)}\right\|, q^{(i+1)}\left\|\alpha q^{(i+1)}\right\|\right\}<\frac{1}{2} .
$$

Главная цель работы - обобщить (0.4) на случай $t=2$. Мы докажем следующие два утверждения.

Теорема 1. Пусть $q \in \Delta\left(\alpha_{1}, \alpha_{2}\right)$ и $q^{\prime}$ - наименьиий знаменатель из $\Delta\left(\alpha_{1}, \alpha_{2}\right)$, для которого выполняются неравенства

$$
q<q^{\prime}, \quad\left\|q^{\prime} \alpha_{1}\right\| \leqslant\left\|q \alpha_{1}\right\|, \quad\left\|q^{\prime} \alpha_{2}\right\| \leqslant\left\|q \alpha_{2}\right\| .
$$

Тогда

$$
\min \left(q\left\|\alpha_{1} q\right\|\left\|\alpha_{2} q\right\|, q^{\prime}\left\|\alpha_{1} q^{\prime}\right\|\left\|\alpha_{2} q^{\prime}\right\|\right)<\frac{1}{2} .
$$

Теорема 2. Пусть $\left(q_{1}, q_{2}\right) \in \Delta^{*}\left(\alpha_{1}, \alpha_{2}\right)$. Выберем из всех знаменателей $\left(q_{1}^{\prime}, q_{2}^{\prime}\right) \in$ $\Delta^{*}\left(\alpha_{1}, \alpha_{2}\right) c$

$$
\left|q_{1}^{\prime}\right| \leqslant\left|q_{1}\right|, \quad\left|q_{2}^{\prime}\right| \leqslant\left|q_{2}\right|, \quad\left\|q_{1} \alpha_{1}+q_{2} \alpha_{2}\right\|<\left\|q_{1}^{\prime} \alpha_{1}+q_{2}^{\prime} \alpha_{2}\right\|
$$

тот, для которого величина $\left\|q_{1}^{\prime} \alpha_{1}+q_{2}^{\prime} \alpha_{2}\right\|$ минимальна. Тогда

$$
\min \left(\left|q_{1} q_{2}\right|\left\|q_{1} \alpha_{1}+q_{2} \alpha_{2}\right\|,\left|q_{1}^{\prime} q_{2}^{\prime}\right|\left\|q_{1}^{\prime} \alpha_{1}+q_{2}^{\prime} \alpha_{2}\right\|\right)<\frac{1}{2} .
$$

По понятным причинам неравенства (0.5) и (0.6) мы назьваем теоремой Валена для двумерных подходящих дробей. Как и в каком виде распространяется этот результат на большие размерности - пока открытьй вопрос.

На самом деле мы сформулируем и докажем более общее утверждение - теорему Валена для трехмерных полных решеток. Теоремы 1 и 2 получаются из нее как следствия. 
1. Относительные минимумы решеток. Пусть $\gamma^{(1)}, \gamma^{(2)}, \gamma^{(3)}$ - произвольный набор линейно-независимых векторов в $\mathbb{R}^{3}$ и

$$
\Gamma=\left\{m_{1} \gamma^{(1)}+m_{2} \gamma^{(2)}+m_{3} \gamma^{(3)} \mid m_{1}, m_{2}, m_{3} \in \mathbb{Z}\right\}
$$

- полная трехмерная решетка. Согласно [1] узел

$$
\gamma \in \Gamma^{\prime}=\Gamma \backslash\{(0,0,0)\}
$$

назьвают относительным минимумом решетки, если параллелепипед

$$
\Pi(\gamma)=\left\{\left(x_{1}, x_{2}, x_{3}\right)|| x_{1}|\leqslant| \gamma_{1}|,| x_{2}|\leqslant| \gamma_{2}|,| x_{3}|\leqslant| \gamma_{3} \mid\right\}
$$

не содержит узлов $\gamma \in \Gamma^{\prime}$, отличных от вершин $\Pi(\gamma)$. Множество всех относительных минимумов решетки Г обозначим через $\mathfrak{M}_{\Gamma}$. Очевидно, что $\gamma$ и $-\gamma$ только одновременно входят в $\mathfrak{M}_{\Gamma}$.

Пусть $\gamma, \gamma^{\prime} \in \mathfrak{M}_{\Gamma}$. Назовем $\gamma^{\prime}$ смежсным с $\gamma$ по координате $j$, если вьполняются условия:

а) $\left|\gamma_{j}\right|<\left|\gamma_{j}^{\prime}\right|$ и $\left|\gamma_{i}^{\prime}\right| \leqslant\left|\gamma_{i}\right|$ для всех $i \neq j$;

б) не существует $\gamma^{\prime \prime} \in \mathfrak{M}_{\Gamma}$, для которого $\left|\gamma_{j}\right|<\left|\gamma_{j}^{\prime \prime}\right|<\left|\gamma_{j}^{\prime}\right|$ и $\left|\gamma_{i}^{\prime \prime}\right| \leqslant\left|\gamma_{i}\right|$ для всех $i \neq j$. Множество всех таких $\gamma^{\prime}$ обозначим через $\mathfrak{M}_{\Gamma}^{(j)}(\gamma)$. Вороной в [1] открыл фундаментальную роль понятия "смежности" для построения алгоритма последовательного вычисления относительных минимумов трехмерных полных решеток, отвечающих чисто вешественньп кубическим расширениям поля рациональных чисел.

Лемма 1. Пусть $\gamma$ и $\gamma^{\prime}$ - относительные минимумы полной решетки $Г$ в $\mathbb{R}^{3}$ $u \gamma^{\prime}$ смехен с $\gamma$ по некоторой координате. Тогда $\gamma$ и $\gamma^{\prime}$ мохнно дополнить некоторым третьим узлом $\gamma^{\prime \prime}$ до базиса Г.

ДокАЗАтЕльство. Ввиду симметрии достаточно рассмотреть случай $\gamma^{\prime} \in \mathfrak{M}_{\Gamma}^{(1)}(\gamma)$. Выберем некоторьй базис $\zeta^{(1)}, \zeta^{(2)}, \zeta^{(3)}$ решетки Г и положим

$$
\gamma=m_{1} \zeta^{(1)}+m_{2} \zeta^{(2)}+m_{3} \zeta^{(3)}, \quad \gamma^{\prime}=n_{1} \zeta^{(1)}+n_{2} \zeta^{(2)}+n_{3} \zeta^{(3)} \text {. }
$$

Пусть

$$
D_{i j}=\operatorname{det}\left(\begin{array}{cc}
m_{i} & m_{j} \\
n_{i} & n_{j}
\end{array}\right)=m_{i} n_{j}-m_{j} n_{i} .
$$

Если мы докажем, что НОД $\left(D_{23}, D_{31}, D_{12}\right)=1$, то найдутся целые $l_{1}, l_{2}, l_{3}$, удовлетворяющие соотношению

$$
l_{1} D_{23}+l_{2} D_{31}+l_{3} D_{12}=1
$$

для которых $\gamma, \gamma^{\prime}, \gamma^{\prime \prime}-$ базис $Г$ с

$$
\gamma^{\prime \prime}=l_{1} \zeta^{(1)}+l_{2} \zeta^{(2)}+l_{3} \zeta^{(3)}
$$

Предположим, что это не так. То есть для некоторого натурального $q \neq 1$

$$
D_{23} \equiv D_{31} \equiv D_{12} \equiv 0 \quad(\bmod q)
$$


или $m_{i} n_{j}-m_{j} n_{i} \equiv 0(\bmod q)$ для всех $i, j \in\{1,2,3\}$. Так как $\gamma, \gamma^{\prime} \in \mathfrak{M}_{\Gamma}$, то

$$
\text { НОД }\left(m_{1}, m_{2}, m_{3}\right)=\text { НОД }\left(n_{1}, n_{2}, n_{3}\right)=1 .
$$

Следовательно, найдется номер $i \in\{1,2,3\}$, а также целые $a$ и $b$, одновременно не равные нулю, для которых

$$
a \equiv n_{i} \quad(\bmod q), \quad b \equiv-m_{i} \quad(\bmod q)
$$

и $-q / 2<a, b \leqslant q / 2$. Поскольку

$$
a \gamma+b \gamma^{\prime}=\left(a m_{1}+b n_{1}\right) \zeta^{(1)}+\left(a m_{2}+b n_{2}\right) \zeta^{(2)}+\left(a m_{3}+b n_{3}\right) \zeta^{(3)}
$$

и для $j=1,2,3$

$$
a m_{j}+b n_{j} \equiv n_{i} m_{j}-m_{i} n_{j} \equiv 0 \quad(\bmod q),
$$

то вектор $\tilde{\gamma}=\left(a \gamma+b \gamma^{\prime}\right) / q-$ узел из $\Gamma^{\prime}$. При этом для $j=1,2,3$

$$
\left|\tilde{\gamma}_{j}\right| \leqslant \frac{1}{2}\left|\gamma_{j}\right|+\frac{1}{2}\left|\gamma_{j}^{\prime}\right|
$$

Отсюда следует, что $\left|\tilde{\gamma}_{1}\right|<\left|\gamma_{1}^{\prime}\right|$ и

$$
\left|\tilde{\gamma}_{2}\right| \leqslant\left|\gamma_{2}\right|, \quad\left|\tilde{\gamma}_{3}\right| \leqslant\left|\gamma_{3}\right| .
$$

Так как $\gamma, \gamma^{\prime} \in \mathfrak{M}_{\Gamma}$ с $\Pi(\gamma) \neq \Pi\left(\gamma^{\prime}\right)$ и $\left|\gamma_{1}\right|<\left|\gamma_{1}^{\prime}\right|$, вьполняется хотя бы одно из неравенств: $\left|\gamma_{2}^{\prime}\right|<\left|\gamma_{2}\right|,\left|\gamma_{3}^{\prime}\right|<\left|\gamma_{3}\right|$. Поэтому из (1.1) следует, что, по крайней мере, одно из неравенств (1.2) можно заменить строгим. Но тогда любой относительный минимум решетки Г из П $(\tilde{\gamma})$ нарушает условие б) в определении смежности $\gamma^{\prime}$ с $\gamma$, и мы пришли к противоречию. Лемма 1 полностью доказана.

ЗАмЕчАниЕ. Эта лемма сформулирована в работе [1], и ее доказательство опущено. При этом Вороной отметил, что ситуация аналогична подробно разобранному им двумерному случаю. Поэтому для удобства читателя мы привели здесь доказательство леммы 1.

Пусть $\sigma=(\sigma(1), \sigma(2), \sigma(3))$ - любая перестановка чисел $(1,2,3)$ и $\Lambda=\left(\lambda_{1}, \lambda_{2}, \lambda_{3}\right)$ произвольный набор вещественных чисел, отличных от нуля. Определим отображение $F_{\Lambda}^{(\sigma)}: \mathbb{R}^{3} \rightarrow \mathbb{R}^{3}$ по правилу

$$
\left(x_{1}, x_{2}, x_{3}\right) \rightarrow\left(\lambda_{1} x_{\sigma(1)}, \lambda_{2} x_{\sigma(2)}, \lambda_{3} x_{\sigma(3)}\right)
$$

которое преобразует решетку $Г$ в решетку $\widetilde{\Gamma}=F_{\Lambda}^{(\sigma)}(\Gamma)$. Из определения непосредственно вытекает следующее утверждение.

Лемма 2. Пусть Г - произвольная решетка в $\mathbb{R}^{3}$. Тогда:

1) $\quad F_{\Lambda}^{(\sigma)}\left(\mathfrak{M}_{\Gamma}\right)=\mathfrak{M}_{\widetilde{\Gamma}}$

2) $\quad F_{\Lambda}^{(\sigma)}\left(\mathfrak{M}_{\Gamma}^{(j)}(\gamma)\right)=\mathfrak{M}_{\widetilde{\Gamma}}^{(\sigma(j))}(\tilde{\gamma}), \quad \tilde{\gamma}=F_{\Lambda}^{(\sigma)}(\gamma)$;

3) $\quad\left|\tilde{\gamma}_{1} \tilde{\gamma}_{2} \tilde{\gamma}_{3}\right|=\left|\lambda_{1} \lambda_{2} \lambda_{3}\right|\left|\gamma_{1} \gamma_{2} \gamma_{3}\right|$. 
2. Теорема Валена для полных трехмерных решеток. Главная цель этого раздела - доказательство следующего результата.

ТеоремА. Пусть Г - полная трехмерная решетка в $\mathbb{R}^{3}$ и $\gamma^{\prime}$ - смежный по некоторой координате относительный минимум $с \gamma \in \mathfrak{M}_{\Gamma}$. Тогда

$$
\min \left\{\left|\gamma_{1} \gamma_{2} \gamma_{3}\right|,\left|\gamma_{1}^{\prime} \gamma_{2}^{\prime} \gamma_{3}^{\prime}\right|\right\}<\frac{1}{2} d(\Gamma)
$$

где $d(\Gamma)>0$ - определитель $Г$.

Если хотя бы одна из координат $\gamma$ или $\gamma^{\prime}$ равна нулю, то оценка (2.1) тривиальным образом верна. Поэтому мы будем считать далее, что $\gamma_{1} \gamma_{2} \gamma_{3} \gamma_{1}^{\prime} \gamma_{2}^{\prime} \gamma_{3}^{\prime} \neq 0$. В силу леммы 2 достаточно рассмотреть случай, когда

$$
\gamma=(1,1,1), \quad \gamma^{\prime}=(\lambda, \mu, \sigma)
$$

c $1<\lambda<\infty, 0<|\mu|,|\sigma| \leqslant 1,|\mu|+|\sigma| \neq 2$.

Лемма 3. Пусть $(\lambda, \mu, \sigma) \in \mathfrak{M}_{\Gamma}^{(1)}(1,1,1)$. Тогда хотя бы одно из чисел $\mu, \sigma$ отрицательно.

ДокАЗАТЕЛЬСТво. Пусть $0 \leqslant \mu, \sigma \leqslant 1$. Расмотрим узел

$$
\gamma^{\prime \prime}=(\lambda, \mu, \sigma)-(1,1,1)=(\lambda-1, \mu-1, \sigma-1) .
$$

Так как $(1,1,1) \in \mathfrak{M}_{\Gamma}$, то $1<\lambda-1$. Но тогда любой относительный минимум из $\Pi\left(\gamma^{\prime \prime}\right)$ нарушает условие б) смежности $(\lambda, \mu, \sigma) \mathrm{c}(1,1,1)$, и мы пришли к противоречию. Лемма 3 доказана.

С учетом этого результата мы далее можем считать, что $(\lambda,-\mu, \sigma) \in \mathfrak{M}_{\Gamma}^{(1)}(1,1,1)$, где

$$
1<\lambda<\infty, \quad 0<\mu \leqslant 1, \quad 0<|\sigma| \leqslant 1, \mu+|\sigma| \neq 2 .
$$

Из леммы 1 следует, что $\gamma$ и $\gamma^{\prime}$ всегда можно дополнить до базиса $Г$ третьим узлом $\gamma^{\prime \prime}=(\theta, \xi, \eta)$. При этом для любых целых $m$ и $n$

$$
\gamma, \gamma^{\prime}, \pm\left(\gamma^{\prime \prime}+m \gamma+n \gamma^{\prime}\right)
$$

- также базис Г. Поскольку сдвиги множества

$$
\left\{\alpha(1,1)+\beta(\lambda,-\mu) \mid-\frac{1}{2}<\alpha, \beta \leqslant \frac{1}{2}\right\}
$$

на узлы двумерной решетки

$$
\{m(1,1)+n(\lambda,-\mu) \mid m, n \in \mathbb{Z}\}
$$

покрьвают всю плоскость $\mathbb{R}^{2}$, мы всегда можем выбрать узел $\gamma^{\prime \prime}=(\theta, \xi, \eta)$ таким, что $|\theta|<\lambda,|\xi| \leqslant 1$. 
Можно предполагать, что $\eta$ - неотрицательное и наименьшее из возможных. В этом случае назовем тройку

$$
\gamma=(1,1,1), \quad \gamma^{\prime}=(\lambda,-\mu, \sigma), \quad \gamma^{\prime \prime}=(\theta, \xi, \eta)
$$

приведенным базисом Г. Заметим, что

$$
d(\Gamma)= \pm \operatorname{det}\left(\begin{array}{ccc}
1 & \lambda & \theta \\
1 & -\mu & \xi \\
1 & \sigma & \eta
\end{array}\right)=|\eta(\lambda+\mu)-\theta(\sigma+\mu)-\xi(\lambda-\sigma)|
$$

Поскольку неравенство $0 \leqslant \eta<1$ нарушает условия

$$
(1,1,1) \in \mathfrak{M}_{\Gamma}^{(1)}, \quad(\lambda,-\mu, \sigma) \in \mathfrak{M}_{\Gamma}(1,1,1),
$$

то всегда

$$
1 \leqslant \eta<\infty, \quad|\theta|<\lambda, \quad|\xi| \leqslant 1 .
$$

Так как $\eta$ выбирается минимальным, то по причине наличия узлов

$$
\begin{aligned}
(\theta, \xi, \eta)-(1,1,1) & =(\theta-1, \xi-1, \eta-1), \\
(\theta, \xi, \eta)+(\lambda,-\mu, \sigma) & =(\lambda+\theta,-\mu+\xi, \sigma+\eta), \\
(\theta, \xi, \eta)-(\lambda,-\mu, \sigma) & =(-\lambda+\theta, \mu+\xi,-\sigma+\eta), \\
(\theta, \xi, \eta)-(1,1,1)-(\lambda,-\mu, \sigma) & =(-1-\lambda+\theta,-1+\mu+\xi,-1-\sigma+\eta), \\
(\theta, \xi, \eta)-(1,1,1)+(\lambda,-\mu, \sigma) & =(-1+\lambda+\theta,-1-\mu+\xi,-1+\sigma+\eta),
\end{aligned}
$$

и получаем дополнительную к (2.6) серию ограничений: в соответствии с $(2.7)$ и $(2.8)$ для $-1 \leqslant \sigma<0$

$$
-1 \leqslant \xi<0
$$

в соответствии с $(2.7)$ и $(2.9)$ для $0<\sigma \leqslant 1$

$$
-\lambda<\theta<0
$$

в соответствии с $(2.8)$ для $-1 \leqslant \sigma<0$ и $-\lambda<\theta<0$

$$
1<\mu-\xi
$$

в соответствии с (2.7) для $0<\sigma \leqslant 1$ и $0 \leqslant \xi \leqslant 1$

$$
-\lambda<\theta \leqslant 1-\lambda
$$

в соответствии с $(2.10)$ для $-1<\sigma<0$

$$
\mu+\xi<0 \text { или } \theta \leqslant 1 \text {; }
$$

в соответствии с (2.11) для $0<\sigma<1$

$$
\xi<\mu \text {. }
$$

Мы докажем для приведенных базисов вида (2.4) решетки Г оценку

$$
\min (1,|\lambda \mu \sigma|)<\frac{1}{2} d(\Gamma)
$$

Теорема, сформулированная в начале раздела, непосредственно следует отсюда с помощью леммы 2 . Для $2<d(\Gamma)<\infty$ оценка (2.18) вьполняется автоматически. Поэтому мы будем рассматривать только случай $0<d(\Gamma) \leqslant 2$ и докажем неравенство (см. (2.5))

$$
2|\lambda \mu \sigma|)<\eta(\lambda+\mu)-\theta(\sigma+\mu)-\xi(\lambda-\sigma)=d(\Gamma) .
$$


(I) Пусть $-1 \leqslant \sigma<0$. В соответствии с $(2.12)$ всегда $-1 \leqslant \xi<0$.

1. Случай $-\lambda<\theta \leqslant 0$ и $\sigma+\mu \geqslant 0$. Тогда

$$
\eta(\lambda+\mu)-\theta(\sigma+\mu)-\xi(\lambda-\sigma)>\lambda+\mu>2 \sqrt{\lambda \mu} \geqslant 2 \lambda \mu \geqslant 2|\lambda \mu \sigma| .
$$

2. Случай $-\lambda<\theta \leqslant 0$ и $\sigma+\mu<0$. С помощью (2.14) находим

$$
\begin{aligned}
& \eta(\lambda+\mu)-\theta(\sigma+\mu)-\xi(\lambda-\sigma)>\lambda+\mu+\lambda(\sigma+\mu)-\xi(\lambda-\sigma) \\
& \quad=\lambda+\mu+\lambda \sigma+\lambda(\mu-\xi)+\xi \sigma \geqslant \lambda+\mu-\lambda+\lambda=\lambda+\mu \geqslant 2|\lambda \mu \sigma| .
\end{aligned}
$$

3. Случай $0<\theta<\lambda$ и $\sigma+\mu \leqslant 0$ очевиден.

4. Случай $0<\theta<\lambda$ и $\sigma+\mu>0$. Если $\theta \leqslant \eta$, то

$$
\eta(\lambda+\mu)-\theta(\sigma+\mu)-\xi(\lambda-\sigma) \geqslant \eta(\lambda+\mu)-\eta(\sigma+\mu)=\eta(\lambda-\sigma)>2|\lambda \mu \sigma| .
$$

Для $1 \leqslant \eta<\theta<\lambda$ с помощью $(2.16)$ находим

$$
\begin{aligned}
& \eta(\lambda+\mu)-\theta(\sigma+\mu)-\xi(\lambda-\sigma)>\lambda+\mu-\lambda(\sigma+\mu)+\mu(\lambda-\sigma) \\
& \quad=\lambda+\mu-\sigma(\lambda+\mu)>\lambda+\mu>2|\lambda \mu \sigma| .
\end{aligned}
$$

5. Случай $0<\theta<\lambda$ и $\sigma+\mu<0$ очевиден.

(II) Пусть $0<\sigma \leqslant 1$. В соответствии с (2.13) всегда $-\lambda<\theta<0$.

1. Случай $\xi \leqslant 0$ очевиден.

2. Случай $\xi>0$.

В соответствии с (2.15) $\lambda+\theta \leqslant 1$. Для $\sigma=1$

$$
\begin{gathered}
\eta(\lambda+\mu)-\theta(1+\mu)-\xi(\lambda-1)=\eta(\lambda+\mu)-(-1+\lambda+\theta)(1+\mu) \\
-(-1+\mu-\xi)(\lambda-1) \geqslant \lambda+\mu>2 \lambda \mu \sigma .
\end{gathered}
$$

Для $0<\sigma<1$ воспользуемся еще и оценкой $\xi<\mu$ из (2.17). Тогда

$$
\begin{gathered}
\eta(\lambda+\mu)-\theta(\sigma+\mu)-\xi(\lambda-\sigma)>\lambda+\mu-\theta \sigma-\theta \mu-\mu \lambda+\mu \sigma \\
\geqslant \lambda+\mu-\mu(\theta+\lambda)+\mu \sigma \geqslant \lambda+\mu \sigma>2 \lambda \mu \sigma .
\end{gathered}
$$

Итак, оценка (2.19), а вместе с ней и теорема доказаны.

Пусть $0<\varepsilon<1$. Нетрудно проверить, что для

$$
\Gamma=\left\{m_{1}(1,1,1)+m_{2}(1+\varepsilon,-1+\varepsilon, 1)+m_{3}(-\varepsilon, 0,1+\varepsilon) \mid m_{1}, m_{2}, m_{3} \in \mathbb{Z}\right\}
$$

$(1,1,1)$ - относительньй минимум и $(1+\varepsilon,-1+\varepsilon, 1) \in \mathfrak{M}_{\Gamma}^{(1)}(1,1,1)$ Кроме того, $d(\Gamma)=$ $2+\varepsilon(4-\varepsilon)$. Отсюда следует, что коэффициент $1 / 2$ в правой части $(2.1)$ нельзя заменить меньшим числом.

Отметим, что двумерный аналог теоремы с оценкой

$$
\min \left\{\left|\gamma_{1}^{\prime} \gamma_{2}^{\prime}\right|,\left|\gamma_{1} \gamma_{2}\right|\right\}<\frac{1}{2} d(\Gamma)
$$

для смежных относительных минимумов $\left(\gamma_{1}, \gamma_{2}\right)$ и $\left(\gamma_{1}^{\prime}, \gamma_{2}^{\prime}\right)$ двумерной полной решетки $\Gamma$ непосредственно следует из результатов работы [1]. 
3. Теорема Валена для подходящих дробей. Пусть $\left(\alpha_{1}, \alpha_{2}\right)$ - пара вещественных чисел. Определим решетку

$$
\Gamma=\left\{m(1,0,0)+n(0,1,0)+q\left(-\alpha_{1},-\alpha_{2}, 1\right) \mid m, n, q \in \mathbb{Z}\right\} .
$$

Рассмотрев смежные относительные минимумы Г по третьей координате, из (2.1) получим теорему 1, поскольку $d(\Gamma)=1$ и элементы $(3.1)$ имеют вид

$$
\left(m-q \alpha_{1}, n-q \alpha_{2}, q\right)
$$

Определим еще одну решетку

$$
\Gamma^{*}=\left\{q_{1}\left(1,0,-\alpha_{1}\right)+q_{2}\left(0,1,-\alpha_{2}\right)+m(0,0,1) \mid q_{1}, q_{2}, m \in \mathbb{Z}\right\} .
$$

Теорема 2 получается из (2.1) для смежных относительных минимумов по третьей координате, поскольку и в этом случае $d\left(\Gamma^{*}\right)=1$.

Следует отметить, что можно доказать еще один вариант теорем 1 и 2 , если для (3.1) и (3.2) рассматривать смежные относительные минимумы по координатам, отличным от третьей.

\section{СПИСОК ЦИТИРОВАННОЙ ЛИТЕРАТУРЫ}

[1] Вороной Г.Ф. Об одном обобщении алгорифмма непрерывных дробей // Собрание сочинений. Т. 1. Киев, 1952. С. 197-391.

[2] Венков Б. А. Элементарная теория чисел. М.: ОНТИ, 1937.

[3] Кассельс Дж. Введение в теорию диофантовых приближений. М.: ИЛ, 1961.

Хабаровское отделение

Института прикладной математики ДВО РАН

Поступило

E-mail: vab@iam.khabarovsk.su

10.08 .98 